\title{
Zwei Jahre EEG - eine Erfolgsbilanz!
}

\author{
Vor gut zwei Jahren, am 1. April 2000, trat das Erneverbare-Energien-Gesetz \\ (EEG) in Kraft. Mit ihm wurde das Ziel verfolgt, den Ausbau der erneverbaren \\ Energien zur Stromerzeugung als ein zentrales Element für Klimaschutz und \\ nachhaltige Entwicklung zu fördern. Das Gesetz hat gewirkt - es hat bei nahezu \\ allen Erneverbaren zu deutlichem Wachstum geführt. Eine ökonomische Detail- \\ analyse zeigt Folgen und Weiterentwicklungsbedarf des Gesetzes auf.
}

D ie Förderung der ernewerbaren Energien (EE) ist nicht nur ein Gebot, das sich aus Gründen des Klimaschutzes und der zur Neige gehenden bzw. zu risikoreichen fossilen und nuklearen Technologien ableitet. Sie entspricht auch den politischen Anforderungen auf nationaler (Klimaschutzprogramm der Bundesregierung), europäischer (EURichtlinie 2001/77/EG) und internationaler Ebene (Kyoto-Abkommen). Für Deutschland besteht das Richtziel der Steigerung des EE-Anteils an der Stromversorgung auf 12,5 Prozent bis 2010. Der deutsche Weg zur Förderung der EE besteht aus einem Maßnahmen-Mix, in dem das zentrale Instrument EEG durch zuschuss- und kreditbasierte Förderprogramme ergänzt wird (1). Das EEG verpflichtet Netzbetreiber, EE-Strom abzunehmen und zu vergüten. Die im Gesetz festgelegten Vergütungssätze sollen einen wirtschaftlichen Betrieb der berücksichtigten EE-Anlagen ermöglichen. Dabei werden primär die so genannten neuen $\mathrm{EE}$ gefördert: Windenergie, Photovoltaik, Bioenergie und geothermische Stromerzeugung, aber auch Wasserkraft bis fünf Megawatt.

Ein vereinfachtes Resümee zu den Wirkungen des EEG kann lauten: Die Hoffnungen auf einen deutlichen Aufschwung wurden in nahezu allen EEBranchen erfüllt. Insbesondere in den Bereichen Windenergie, Photovoltaik und Bioenergie erfolgte eine deutliche Zubausteigerung. Dank des EEG konnte der Anteil der EE am deutschen Bruttostromverbrauch von 5,6 Prozent im Jahr 1999 auf 7 Prozent 2001 und voraussichtlich 7,8 Prozent 2002 gesteigert werden. Setzt man demgegenüber eine idealtypische Wachstumskurve von 1999 bis zum Zielwert 12,5 Prozent EE-Strom 2010 an, dann konnte der daraus resultierende Zielwert von beispielsweise 6,5 Prozent für 2001 bereits übertroffen werden.
Zur Beurteilung des Erfolgs sind jedoch tiefergehende Analysen vorzunehmen. Es ist zu beantworten, wie sich die Märkte im Detail entwickelt haben, ob und in welchen Bereichen die Vergütungen ausreichend oder gar zu hoch angesetzt waren, ob die Anlagen mit den perspektivisch größten Potenzialen gefördert wurden etc. Das Institut für ökologische Wirtschaftsforschung untersuchte in einem Gutachten im Auftrag des Bundeswirtschaftsministeriums daher bei allen betroffenen EE detailliert die Marktund Kostensituationen (2). Damit wurde auch ein Beitrag zur Verbesserung der derzeit noch überwiegend schlechten Datenlage geleistet. Bei der Bestimmung der Stromgestehungskosten ergaben sich sehr große Spannbreiten. Es wurden jeweils für verschiedene Leistungsgrößenklassen sowie weitere spezifische Differenzierungsgrößen wie etwa standortrelevante Faktoren bei Wind- und Wasserkraft oder Brennstoffarten bei Bioenergie Durchschnittswerte ermittelt und zusätzlich Variationen zentraler Parameter vorgenommen. Danach zeigte sich, dass in allen Bereichen die größten Anlagenklassen die niedrigsten Gestehungskosten erzielen: allen voran die Wasserkraft, aber beispielsweise auch 20 MegawattAltholz-Anlagen. Als weiteres allgemeines Ergebnis kann festgehalten werden, dass in allen Bereichen außer der Photovoltaik einzelne Anlagenklassen unter den gegenwärtigen Förderbedingungen wirtschaftlich betrieben werden können. Dies gilt allerdings wie erwähnt im Wesentlichen nicht für mittlere und kleinere Anlagen, die in Bereichen wie der Bioenergie die größeren Potenziale bergen und darïber hinaus aufgrund besserer Wärmenutzungsmöglichkeiten auch aus Effizienzgesichtspunkten häufig vorteilhafter sind.

Diese Zusammenhänge sowie einige Spezifika der einzelnen neuen EE werden nachfolgend kurz erläutert.

\section{- Windenergiewachstum auf hohem Niveau}

Die Windenergie ist nach absoluten Zahlen die bedeutendste EE-Wachstumsbranche. Dem beeindruckenden Zubau der letzten Jahre stand aufgrund des windschwachen Jahres 2001 ein deutlich geringerer Anstieg der erzeugten Strommenge gegenüber (vgl. Abbildung). Die Stromgestehungskosten von Windkraftanlagen werden entscheidend durch die Standortqualität beeinflusst. Derzeit werden auch viele Standorte ab 1.500 Volllaststunden erschlossen. Die berechneten Gestehungskosten weisen darauf hin, dass geringfügige Anpassungen bei der Gestaltung der Einspeisevergütungen in Abhängigkeit von der Standortgüte zusammen mit der bereits seit diesem Jahr wirksamen jährlichen Absenkung der

\section{Abb. 1: Gesamte EE-Stromproduktion und Anteil am Bruttostromverbrauch}

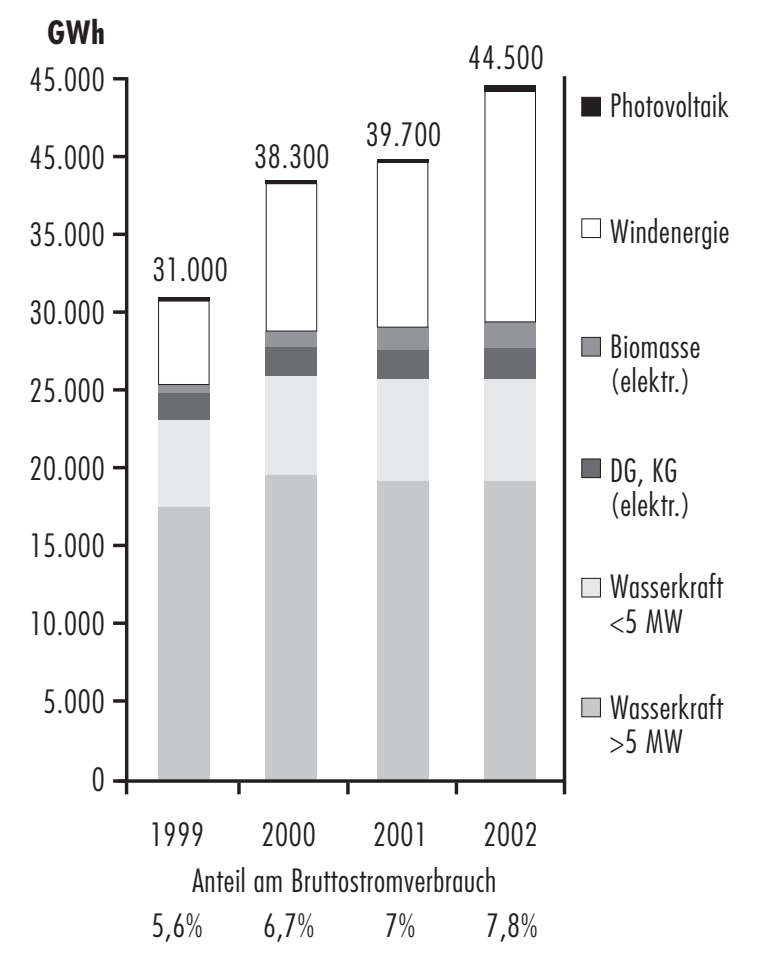

Quelle: eigene Darstellung 
Vergütungssätze für Neuanlagen um 1,5 Prozent dazu beitragen könnten, weitere Kostenreduktionen über die bereits erreichten zu stimulieren.

\section{- Kleine Bioenergieanlagen noch unwirtschaftlich}

Bei der Bioenergie ist bislang die Stromerzeugung aus Holz und aus Biogas am bedeutsamsten. Das EEG hat in Verbindung mit dem Marktanreizprogramm insbesondere beim Biogas einen deutlichen Investitionsschub ausgelöst: 2001 hat sich die Anlagenzahl um mehr als 50 Prozent gesteigert, die installierte Leistung hat sogar um mehr als 100 Prozent auf 140 Megawatt zugenommen.

Die Wirtschaftlichkeit von Biogasanlagen wird entscheidend von der Anlagengröße, der Prozesseffizienz und den Kosten für Einsatzstoffe bestimmt. Stromgestehungskosten in Höhe der EEGVergütungssätze lassen sich zur Zeit nur in größeren Biogasanlagen erzielen. Unter Einbeziehung der Förderung aus dem Marktanreizprogramm liegen die Kosten bis zu einer Leistung von 50 Kilowatt bei etwa $14 \mathrm{Cent} / \mathrm{kWh}$ und bei 100 Kilowatt bei etwa 12 Cent/kWh. Daher empfiehlt sich eine Erhöhung der Vergütungssätze für den kleinen Leistungsbereich. Wird das Biogas zu wesentlichen Anteilen aus nachwachsenden Rohstoffen erzeugt, erhöhen sich die Gestehungskosten durch den zusätzlich anfallenden Anbau- und Ernteaufwand.

Holz wird zur Zeit in Anlagen mit einer installierten elektrischen Leistung von insgesamt etwa 150 Megawatt genutzt. Aufgrund der unsicheren Entwicklungen auf dem Brennstoffmarkt sind bislang kaum neue Anlagen entstanden. Die Kosten der Holzbrennstoffe unterscheiden sich je nach Herkunft stark. Auch die Leistungsgröße beeinflusst die Wirtschaftlichkeit. Tendenziell erreichen größere Anlagen günstigere Stromgestehungskosten, unterliegen dafür jedoch einem größeren Beschaffungsproblem. Wichtige unerschlossene Potenziale bestehen beim Waldrestholz, dieses ist jedoch der teuerste Brennstoff und sein Einsatz bislang unwirtschaftlich. Hier könnte eine brennstoffspezifische Erhöhung der Vergütung Investitionsanreize setzen.

\section{- Photovoltaik: schnelles politisches Handeln gefragt}

Die Photovoltaik (PV) ist zwar bislang der kleinste und teuerste Bereich, weist jedoch die größten Zuwachsraten auf. Dabei hat erst die Kombination des 100.000-Dächer-Programms mit dem EEG den Durchbruch für die Branche gebracht: Seit 2000 wird in deutlich höherem Ausmaß in der PV-Industrie investiert. Dazu sind Konzentrationsprozesse und internationale Verflechtungen des bislang importabhängigen Marktes zu verzeichnen. Die getätigten und geplanten Investitionen werden aller Voraussicht nach dazu führen, dass die deutsche PV-Nachfrage bereits ab dem nächsten Jahr durch die Inlandsproduktion gedeckt werden könnte. Zusammen mit der gegenwärtigen Weltmarktdominanz der japanischen Konkurrenz, die sich bereits in einer fortgeschritteneren Massenproduktionsphase befindet, bedeutet dies einerseits eine Gefahr für die deutsche Industrie, die bislang eine sehr niedrige Exportquote aufweist, andererseits können aus dieser Marktsituation deutliche Kostendegressionen resultieren - wahrscheinlich einhergehend mit drastischen Marktbereinigungen. Kostendegressionen sind angesichts der gegenwärtigen Vergütungen auch notwendig, denn noch sind nur die größten Anlagen annähernd wirtschaftlich zu betreiben - die Masse der kleineren Hausdachanlagen jedoch nicht. Der allgemeine Durchschnittswert für 2001 lag unter Berücksichtigung des 100.000-Dächer-Kredits bei 56 Cent/ kWh. Als weiteres Damokles-Schwert schwebt über der PV-Branche das Erreichen des $350 \mathrm{Me}$ gawatt-Deckels gesamter installierter Leistung Ende 2003, der im Förderprogramm und EEG festgeschrieben ist. Die PV-Marktanalyse unterstreicht somit, dass die dynamischen Entwicklungen ein möglichst schnelles politisches Handeln erfordern, sowohl in Bezug auf eine Anschlussförderregelung als auch auf eine Exportförderstrategie, damit keine politisch induzierten stranded investments in der deutschen PV-Wachstumsbranche entstehen.

\section{Geothermie vor dem Take-off}

Eine neue, viel versprechende Technologie stellt die geothermische Stromerzeugung dar. Derzeit sind noch keine solchen Anlagen in Deutschland realisiert, mit der Einführung des EEG haben sich die Projekte und die Zahl der Akteure in diesem Feld jedoch stark erhöht. Die erstmalige breite Erhebung vieler Daten der aktuell laufenden Projekte und Verdichtung zu einer empirisch basierten Kostenschätzung ergab, dass derartige Anlagen voraussichtlich noch nicht wirtschaftlich darstellbar sind. Dies wird durch die besondere Risikostruktur und den bestehenden Forschungsbedarf und -anteil verstärkt. Die Förderung von Letzterem zum Beispiel durch die Mittel des Zukunftsinvestitionsprogramms der Bundesregierung deckt in vielen
Fällen die Finanzierungslücken - und stellt gleichzeitig die erforderliche Wissensgenerierung und Technologieentwicklung sicher. Angesichts der noch hohen Erfolgsrisiken werden perspektivisch eher kleinere Anlagen realisiert werden, trotz höherer spezifischer Kosten. Im Falle einer sich abzeichnenden derartigen Entwicklung ist zu einem späteren Zeitpunkt eine höhere Vergütung für kleinere Anlagen zu erwägen.

\section{$\checkmark$ Fazit}

Aufgrund der aufgezeigten Entwicklungen kann das EEG gemessen an den Zielsetzungen als Erfolg in allen EE-Sparten gewertet werden - und das, obwohl nur in vergleichsweise geringem Ausmaß über alle EE und Anlagengrößen bereits Wirtschaftlichkeit erreicht werden konnte. Die in vielen Bereichen erzielte deutliche Verbesserung reichte als Signal für viele Investoren aus. Dabei sollte berücksichtigt werden, dass zu Beginn einer sprunghaften Wachstumsphase nicht mit starken Kostensenkungen gerechnet werden kann; diese setzen erst mit entsprechender Verzögerung ein, wenn die Branchen in massiven Kapazitätsaufbau einsteigen und gleichzeitig ein hohes Innovationspotenzial vorhanden ist (3). Der tiefere Blick in die Branchen- und Technologieentwicklungen offenbart geringfügigen, jeweils spezifischen Anpassungsbedarf der EEG-Vergütungsregelungen, der allerdings sogar im Rahmen kostenneutraler Umschichtungen bewerkstelligt werden könnte.

\section{Anmerkungen}

(1) Vgl. zu genereller Bilanz und Perspektiven der bisherigen Förderpolitik auch das Spezial "Energiewende in Sicht" in der Ausgabe 3-4/01 von Ökologisches Wirtschaften.

(2) Die Bereiche Windenergie und Wasserkraft wurden dabei vom Kooperationspartner Institut für solare Energietechnik (ISET), Kassel bearbeitet. Die Ergebnisse werden zentraler Bestandteil des EEG-Erfahrungsberichts des Wirtschaftsministeriums am 30. Juni 2002 an den Deutschen Bundestag sein. Eine Veröffentlichung der Studie ist in Vorbereitung. (3) Vgl. dazu am Beispiel der Solarenergie ausführlich Hirschl, B.: Mehr Geduld mit dem Boom. In: Umwelt kommunale ökologische Briefe, Nr. 6 vom 13.3.2002.

\section{Die Autorlnnen}

Bernd Hirschl und Esther Hoffmann sind wissenschaftliche MitarbeiterInnen am Institut für ökologische Wirtschaftsforschung

Kontakt: IÖW, Potsdamer Str. 105, 10785 Berlin,

Tel. 030/ 884594-26, Fax 030/ 8825439,

E-Mail: bernd.hirschl@ioew.de 
(c) 20I0 Authors; licensee IÖW and oekom verlag. This is an article distributed under the terms of the Creative Commons Attribution Non-Commercial No Derivates License (http://creativecommons.org/licenses/by-nc-nd/3.o/), which permits unrestricted use, distribution, and reproduction in any medium, provided the original work is properly cited. 